\title{
Serum 25-hydroxyvitamin D Insufficiency in B-Chronic Lymphoid Leukemia at the Time of Disease Presentation in Pakistan
}

\author{
Saira Parveen*, Rozina Zeeshan, Sadia Sultan, Syed Mohammad Irfan
}

\begin{abstract}
Background: Serum 25-hydroxyvitamin D insufficiency is very common in Pakistan and is often related to inferior prognosis in some cancers but limited data exist for hematopoietic malignancies. This study was conducted to determine the vitamin D insufficiency in B-chronic lymphoid leukemia (CLL) cases at the time of presentation and its possible correlation with clinical staging, hematological parameters and biochemical markers. Materials and Methods: This descriptive cross sectional study was carried at Liaquat National Hospital from January 2011 to June 2013. Sixty patients with B-chronic lymphoid leukemia were enrolled. Complete blood count, vitamin D levels, serum urea, creatinine, uric acid and LDH levels were assessed. Data were compiled and analyzed using SPSS version 21. Results: Out of 60 patients, $42(70 \%)$ were male and $18(30 \%)$ were female. Mean age was 59.0 \pm 9.2 years. The frequency of vitamin $D$ insufficiency was found to be $56.7 \%$. Overall insufficiency was more frequently seen in male gender $(\mathbf{4 0 \%})$. Vitamin $D$ insufficiency demonstrated a positive association with low lactate dehydrogenase levels $(P=0.005)$. No links were established with age, clinical stage, hematological and other biochemical markers. Conclusions: Vitamin D insufficiency is high compared with Western studies. Whether normalization of vitamin D insufficiency in deficient B-CLL patients could improve the clinical outcome or delay disease progression will require further studies.
\end{abstract}

Keywords: B-Chronic lymphoid leukemia - Vitamin D insufficiency

Asian Pac J Cancer Prev, 16 (14), 5983-5986

\section{Introduction}

Chronic lymphoid leukemia (CLL) is the most frequent type of adult leukemia in the western countries with an incidence of 5 individuals per 100,000 annually (Xu et al., 2009; Rozman et al., 1995; Zwiebel et al., 1998). It's a disease that affects mainly people of advanced age but around $20 \%$ of patients are younger than 50 years (Mauro et al., 1999; Zeeshan et al., 2015). CLL is a heterogeneous disease at both clinical and molecular levels with a variable clinical course (Mozaheb et al., 2012; Zeeshan et al., 2015).

Different prognostic factors are involved in decisions making regarding initiation of treatment, predicting the disease outcome and median overall survival ( $\mathrm{Li}$ et al., 2008). Various markers including ZAP-70, molecular studies and mutational status are useful prognostic indicators for disease course and overall survival (Sagatys and Zhang, 2012; Zeeshan et al., 2015). Recently vitamin $\mathrm{D}$ insufficiency has been emerging as a new independent poor prognostic factor in patients with B-CLL (Aref et al., 2013).

Serum vitamin D insufficiency is frequent globally and studies revealed that low levels are often linked with elevated cancer incidence (Shanafelt.et al., 2011). Several reports revealed that low serum $25(\mathrm{OH}) \mathrm{D}$ levels might be associated with amplified incidence of colorectal carcinoma, breast tumors and other malignancies (Yin et al., 2009; Chen et al., 2010; Garland et al., 2009). Furthermore vitamin D insufficiency is also related to inferior prognosis in some malignancies but little data exist for chronic lymphoid leukemia (Molica etal., 2012). Data from one large prospective cohort study suggested that low serum $25(\mathrm{OH}) \mathrm{D}$ levels are associated with inferior time to treatment (TTT) and decreased overall survival (OS) in CLL patients (Shanafelt.et al., 2011).

So far CLL is not curative; identification of reversible/ treatable factors that relate to disease progression or with overall survival would be valuable. Such treatable causes might improve the clinical outcome and may delay disease progression by virtue of replenishment therapy.

The aims of this study were two-fold; primarily to determine the prevalence of vitamin D insufficiency in newly diagnosed patients with CLL and secondarily to correlate insufficiency with clinical staging, hematological parameters and biochemical markers. 


\section{Nquateriafis atrod Methods}

This is a cross sectional prospective study conducted from January 2011 till June 2013. A total of 60 patients with B-chronic lymphoid leukemia were enrolled in the study. All patients were registered in hematology clinic where the record was maintained including history, physical examination and regular follow ups. An informed consent was obtained from all the participating patients.

Diagnosis of B-CLL was established by immuno phenotyping either by immunohistochemistry or by flow cytometry. Patients with other acute or chronic lymphoproliferative disorders, post chemotherapy/ radiotherapy, having non hematopoietic malignancy or with relapsed/ refractory CLL were excluded. Patients who have been on vitamin D supplementation were also excluded.

Venous blood samples were taken for complete blood count, vitamin D levels, serum lactate dehydrogenase, serum urea, creatinine and uric acid. Hematological parameters were determined by Cell Dyne Ruby (Abott, USA). Biochemical markers including serum urea, creatinine, uric acid and LDH were detected by HITACHI 912 (Japan) by photometric assay while serum 25hydroxyvitamin D levels was detected by Hitachi E41 (Japan) by chemiluminescence technique. Diagnosis of vitamin D insufficiency was established at levels $<30 \mathrm{ng} /$ $\mathrm{ml}$. Ethical approval was given by ethical and research committee of Liaquat National Hospital, obtained prior to the study.

Statistical analysis: Statistical analysis was carried out using SPSS version 21. Qualitative data were presented in terms of frequencies and percentages. Mean and standard deviation were reported for quantitative variables. Independent sample t-test and chi-square test of independence were used to identify significant differences and associations between vitamin D levels and study variables.

\section{Results}

A total of 60 confirmed chronic lymphoid leukemia patients using the non probability consecutive sampling were included in this study. $42(70 \%)$ were males and $18(30 \%)$ were females. Male to female ratio were 2.1:1.

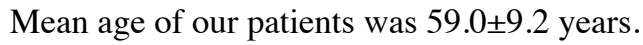

The mean hemoglobin level was $10.8 \pm 2.4 \mathrm{~g} / \mathrm{dl}$ while mean TLC count was $91.5 \pm 87.8 \times 10^{9} / 1$. The mean platelets counts were $197.8 \pm 103.2 \times 10^{\%} / 1$. Evaluated hematological and biochemical markers are shown in table-1.

Serum vitamin D was found to be insufficient in 34 $(56.7 \%)$ patients, while remaining $26(43.3 \%)$ patients were non deficient. The frequency of deficiency is high in male gender, detected in $24(40 \%)$ male patients while $10(16.6 \%)$ females were vitamin D deficient. The comparative analyses of deficient and non deficient patients are shown in table-2.

Mean ages of vitamin D deficient and non deficient patients are statistically not significant $(\mathrm{P}>0.05)$. However comparative analysis revealed statistically significantly low serum Lactate dehydogenase levels of $318.38 \pm 92.79$ in vitamin D deficient patients group as compared with
Table 1. Descriptive Statistics

\begin{tabular}{|c|c|c|c|}
\hline \multicolumn{2}{|l|}{ Parameters } & Mean & $(\mathrm{SD})$ \\
\hline \multicolumn{2}{|l|}{ Age } & 59.0 & $(9.2)$ \\
\hline \multicolumn{2}{|l|}{ Hemoglobin } & 10.8 & $(2.4)$ \\
\hline \multicolumn{2}{|c|}{ Total Leucocyte Count } & 91.5 & $(87.8)$ \\
\hline \multicolumn{2}{|c|}{ Platelet Count } & 197.8 & $(103.2)$ \\
\hline \multicolumn{2}{|c|}{ Absolute Lymphocyte Count } & 91.88 & $(69.43)$ \\
\hline \multicolumn{2}{|c|}{ Serum urea } & 46.2 & $(10.4)$ \\
\hline \multicolumn{2}{|c|}{ Serum creatinine } & 1.29 & $(0.56)$ \\
\hline \multicolumn{2}{|c|}{ Serum Lactate dehydrogenase } & 512 & $(232.61)$ \\
\hline \multicolumn{2}{|c|}{ Serum Uric Acid } & 5.59 & $(2.47)$ \\
\hline & & Frequency & $(\%)$ \\
\hline \multirow[t]{2}{*}{ Gender } & Male & 42 & $(70 \%)$ \\
\hline & Female & 18 & $(30 \%)$ \\
\hline \multirow[t]{2}{*}{ Vitamin D } & Deficient & 34 & $(56.7 \%)$ \\
\hline & Non deficient & 26 & $(43.3 \%)$ \\
\hline \multirow[t]{5}{*}{ Rai-Staging } & Stage 0 & 7 & $(11.6 \%)$ \\
\hline & Stage 1 & 8 & $(13.3 \%$ \\
\hline & Stage 2 & 16 & $(26.7 \%)$ \\
\hline & Stage 3 & 16 & $(26.7 \%)$ \\
\hline & Stage 4 & 13 & $(21.7 \%)$ \\
\hline
\end{tabular}

Table 2. Comparative Analysis of Vitamin D deficient and Non-deficient Patients

\begin{tabular}{|c|c|c|c|}
\hline \multirow[t]{2}{*}{ Parameters } & \multicolumn{2}{|c|}{ Vitamin D Deficiency } & \multirow[t]{2}{*}{ P-value } \\
\hline & $\begin{array}{c}\text { Yes } \\
(n=32)\end{array}$ & $\begin{array}{c}\text { No } \\
(n=24)\end{array}$ & \\
\hline Age & $60.47(9.54)$ & $59.46(11.91)$ & 0.71 \\
\hline Hemoglobin & $10.47(2.90)$ & $11.77(1.63)$ & 0.03 \\
\hline \multicolumn{4}{|c|}{ Total Leucocyte Count } \\
\hline & $107.18(92.20)$ & $68.68(62.72)$ & 0.08 \\
\hline \multicolumn{4}{|c|}{ Absolute lymphocytic count } \\
\hline & $100.04 \pm 75.68$ & $86.16 \pm 59.42$ & 0.7 \\
\hline Platelet Count & 209(104.52) & $203.25(122.13)$ & 0.85 \\
\hline \multicolumn{4}{|c|}{ Serum Lactate dehydrogenase } \\
\hline & $318.38(92.79)$ & $551.50(229.22)$ & 0.005 \\
\hline Serum Creatinine & $1.18(0.49)$ & $1.21(0.42)$ & 0.84 \\
\hline Serum Uric Acid & $5.97(3.65)$ & $4.7(0.83)$ & 0.17 \\
\hline
\end{tabular}

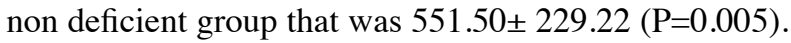
No significant associations were established with clinical stage of disease and hematological markers $(\mathrm{P}>0.05)$.

\section{Discussion}

Chronic lymphoid leukemia is the most frequent type of adult's leukemia that predominantly arises from B-cell lymphocytes (Kermani et al., 2007). Generally CLL is considered as incurable disease. CLL treatment mainly focuses on controlling the disease and its symptoms rather than to cure. Thus it's really worth effective to determine the remediable factors that relate to disease progression in this non curative disease to slow down the disease progression. Recently vitamin D insufficiency has been emerging as a first potentially modifiable host factor associated with poor prognosis in newly diagnosed B-CLL patients (Shanafelt.et al., 2011).

Serum 25-hydoxyvitamin D insufficiency is common globally and approximately $25 \%-50 \%$ of patients seen in routine clinical practice have vitamin D insufficiency and it is estimated that up to 1 billion people worldwide have vitamin D insufficiency (Holick, 2007). As far as locally, 
vitamin D deficiency is concerned only few reports are available; studies were conducted on pediatric age group, pregnant and lactating females and adult's individuals. But we could not found any study exclusively on elderly patients.

One population-based, double-blind, randomized trial found that women who increased their daily vitamin D intake by 1100 IU had reduced risk of cancer by 60\%-77\% (Lappe et al., 2007). The relationship between vitamin D levels and solid tumor risk is well established but far less is known about the relationship between vitamin D and the risk of hematological malignancies.

To date, there have been a handful studies in the literature on this aspect. To the best of our knowledge, this is the first report from our part of the world. The present study has shown vitamin D insufficiency seen in $56.7 \%$ of Pakistani B-CLL patients. In the available literature, we did not come across any Asian study on this complication for comparison with our findings.

When compared with earlier reports, study published from USA revealed $30.5 \%$ of patients being vitamin D deficient in a large prospective cohort study (Shanafelt.et al., 2011). Author concluded that vitamin D insufficiency is associated with inferior time to treatment (TTT) and decreased overall survival (OS) in CLL patients (Shanafelt.et al., 2011).

However the prevalence was quite high in Italian study that disclosed $82.2 \%$ vitamin D deficiency in stage A disease in CLL patients (Molica et al., 2012).

The dissimilarity in frequency of vitamin D insufficiency between us and west could be attributed to difference in environmental factor, ethnic background and dietary habits between different populations.

Vitamin D is not solely a vitamin but a multifunctional pro-hormone and has widespread functions (Feldman et al., 2014). Upcoming studies have demonstrated that $25(\mathrm{OH}) \mathrm{D}$ also has some effects on cellular proliferation, differentiation and apoptosis (Wang et al., 2015). Łuczyńska established that higher concentrations of $25(\mathrm{OH}) \mathrm{D}$ are associated with a reduced risk of CLL (Łuczyńska et al., 2013). Conversely Vitamin $\mathrm{D}$ insufficiency has also been associated with disease progression in CLL (Łuczyńska et al., 2013). Vitamin D3 analog has been shown to induce apoptosis invitro in CLL cells via p53-independent mechanism (Pepper et al., 2003).

In accordance to our findings, prior study from USA disclosed no significant correlation of vitamin D insufficiency with absolute lymphocytic count and advanced disease (Rai stage) in the 229 patient's cohort (Shanafelt.et al., 2011). This is in concurrence to our findings as no correlation was established with absolute lymphocytic count and Rai clinical stage in the present study $(\mathrm{P}>0.05)$.

Large Meta analyses reported from China suggest that low serum $25(\mathrm{OH})$ D levels are significantly associated with poorer prognosis in hematological malignancies (Wang et al., 2015). Aref et al from Egypt also disclosed in a multivariate analysis that $25(\mathrm{OH}) \mathrm{D}$ insufficiency is an independent poor prognostic factor in B-CLL patients (Aref et al., 2013).
Study from France revealed that lower levels of serum $25(\mathrm{OH}) \mathrm{D}$ related to progressive stage of the disease and poor response to therapy and thus to the aggressiveness of the disease (Thomas et al., 2011). Molica et al from Italy identified that $25(\mathrm{OH}) \mathrm{D}$ levels may be an important host factor influencing time to first treatment (TFT).

The importance of vitamin D in CLL is also highlighted by an unexpected observation of a 13-month remission of CLL after the administration of cholecalciferol in an elderly patient with Vitamin D deficiency (Arlet et al., 2012). One previous study of 34 patients with low-grade NHL treated with a synthetic analog of $1,25(\mathrm{OH}) 2 \mathrm{D}$ reported tumor regression in $24 \%$ of patients (Raina et al., 1991). Whether normalizing vitamin D levels may delay disease-progression of such patients will require further testing (Molica etal., 2012).

We would like to mention limitations of our study. Firstly the sample size in our study is small.A large sample would be a better indicator of vitamin D insufficiency in our population. Second limitation is related to observational nature and lack of a control group for comparison. Despite the limitations mentioned above, strength of this study is the fact that this is first local study reported. Thus it provides essential informative data for future prospective studies.

In conclusion, $25(\mathrm{OH}) \mathrm{D}$ insufficiency is not uncommon in B-CLL. It is emerging as a new poor prognostic marker irrespective of clinical stage of disease. Future prospective studies are definitely required to validate this finding. However more beneficial will be a randomized interventional trials on large cohort to determine the delay in disease progression, improvement in clinical outcome and overall survival.

\section{Acknowledgements}

The authors are grateful to the patients who have participated in this study. We thank staff of the Hematology Division of Liaquat National Hospital, for their excellent support.

\section{References}

Aref S, Ibrahim L, Azmy E (2013). Prognostic impact of serum 25-hydroxivitamin $\mathrm{D}[25(\mathrm{OH}) \mathrm{D}]$ concentrations in patients with lymphoid malignancies. Hematol, 18, 20-5.

Arlet JB, Callens C, Hermine O, et al (2012). Chronic lymphocytic leukaemia responsive to vitamin D administration. $\mathrm{Br} J$ Haematol, 156, 148-9.

Chen P, Hu P, Xie D, et al (2010). Meta-analysis of vitamin D, calcium and the prevention of breast cancer. Breast Cancer Res Treat, 121, 469-77.

Feldman D, Krishnan AV, Swami S, Giovannucci E, Feldman $\mathrm{BJ}$ (2014). The role of vitamin D in reducing cancer risk and progression. Nat Rev Cancer, 14, 342-57.

Garland CF, Gorham ED, Mohr SB, Garland FC. (2009). Vitamin $\mathrm{D}$ for cancer prevention: global perspective. Ann Epidemiol, 19, 468-83.

Holick MF (2007). Vitamin D deficiency. N Engl J Med, 357, 266-81.

Li ZJ, Qiu LG, Wu T, et al (2008). The clinical and laboratory features of 263 cases of chronic lymphocytic leukemia. Zhonghua Xue Ye Xue Za Zhi, 29, 300-3 


\section{Saira Parveen et al}

Lappe JM, Travers-Gustafson D, Davies KM, Recker RR, Heaney RP (2007). Vitamin D and calcium supplementation reduces cancer risk: results of a randomized trial. Am J Clin Nutr, 85, 1586-91.

Łuczyńska A, Kaaks R, Rohrmann S, et al (2013). Plasma 25-hydroxyvitamin D concentration and lymphoma risk: results of the European Prospective Investigation into Cancer and Nutrition. Am J Clin Nutr, 98, 827-38.

Mauro FR, Foa R, Giannarelli D, et al (1999). Clinical characteristics and outcome of young chronic lymphocytic leukemia patients: a single institution study of 204 cases. Blood, 94, 448-54.

Molica S, Digiesi G, Antenucci A, et al (2012). Vitamin D insufficiency predicts time to first treatment (TFT) in early chronic lymphocytic leukemia (CLL). Leuk Res, 36, 443-7.

Mozaheb Z, Hasanzadeh N, Abadi MH, Aghaee MA (2012). Chronic lymphocytic leukemia and prognostic factors. Asian Pac J Cancer Prev, 13, 3009-13.

Pepper C, Thomas A, Hoy T, et al (2003). The vitamin D3 analog EB1089 induces apoptosis via a p53-independent mechanism involving p38 MAP kinase activation and suppression of ERK activity in B-cell chronic lymphocytic leukemia cells in vitro. Blood, 101, 2454-60.

Raina V, Cunningham D, Gilchrist N, Soukop M (1991). Alfacalcidol is a nontoxic, effective treatment of follicular small-cleaved cell lymphoma. Br J Cancer, 63, 463-5.

Rozman C, Montserrat E (1995). Chronic lymphocytic leukemia. $N$ Engl J Med, 1333, 1052-57.

Sagatys EM, Zhang L (2012). Clinical and laboratory prognostic indicators in chronic lymphocytic leukemia. Cancer Control, 19, $18-25$.

Shanafelt TD, Drake MT, Maurer MJ, et al (2011). Vitamin D insufficiency and prognosis in chronic lymphocytic leukemia. Blood, 117, 1492-8.

Thomas X, Chelghoum Y, Fanari N, Cannas G (2011). Serum 25-hydroxyvitamin D levels are associated with prognosis in hematological malignancies. Hematol, 16, 278-83.

Wang W, Li G, He X, et al (2015). Serum 25-hydroxyvitamin $\mathrm{d}$ levels and prognosis in hematological malignancies: a systematic review and meta-analysis. Cell Physiol Biochem, 35, 1999-2005.

Xu W, Li JY, Miao KR, et al (2009). The negative prognostic significance of positive direct antiglobulin test in Chinese patients with chronic lymphocytic leukemia. Leuk Lymphom, 50, 1482-7.

Yin L, Grandi N, Raum E, et al (2009). Meta-analysis: longitudinal studies of serum vitamin $\mathrm{D}$ and colorectal cancer risk. Aliment Pharmacol Ther, 30, 113-25.

Zeeshan R, Sultan S, Irfan SM, Kakar J, Hameed MA (2015). Clinico-hematological profile of patients with B-chronic lymphoid leukemia in Pakistan. Asian Pac J Cancer Prev, 16, 793-6.

Zeeshan R, Irfan SM, Sultan S, Bhimani S (2015). ZAP-70 protein expression in B-cell Chronic Lymphoid Leukemia: A single center experience from Pakistan. Asian Pac J Cancer Prev, 16, 1587-90.

Zwiebel JA, Cheson BD (1998). Chronic lymphocytic leukemia: staging and prognostic factors. Semin Oncol, 25, 42-59. 